\title{
Application of Multi-Fractal Detrended Fluctuation Analysis
}

\author{
Rui Yang ${ }^{1, a}$, Xiangyang $\mathrm{Li}^{1, b}$ and Junfeng $\mathrm{Qi}^{2, \mathrm{c}}$ \\ ${ }^{1}$ School of Management, Harbin Institute of Technology, Harbin 150001, China; \\ ${ }^{2}$ China Banking Regulatory Commission, Heilongjiang Authority, Harbin 150001, China; \\ ayangrui49@163.com, bxiangyangli50@163.com, ${ }^{\mathrm{c}} 112862540 @ q q . c o m$
}

\begin{abstract}
Keywords: Multi-Fractal, Detrended Fluctuation Analysis.
\end{abstract}
\begin{abstract}
This paper selects several major stock indices from Europe, America and Asia, and analyze their data from September 1, 2006 to September 30, 2013 based on the MF-DFA (Multi-Fractal Detrended Fluctuation Analysis) method, carry out Hurst exponent and multi-fractal spectrum analysis respectively, and revealed the presence of multi-fractal properties of all the indices, besides, the multi-fractal properties of indices from different continents are affected by different factors. The Hurst exponent calculation shows that Hong Kong Hang Seng Index has the greatest risk among all the major Asian indices, Dow Jones index has the greatest risk among all the major American indices, and the FTSE 100 index has the greatest risk among all the major European indices.
\end{abstract}

\section{Introduction}

In recent years, the MF-DFA method is widely used in researches for financial markets. Feng Ma, et al.[1] investigate the cross-correlations between the stock market in China and markets in Japan, South Korea and Hong Kong based on MF-DFA method, confirm the existence of cross-correlations, and find that the cross-correlations of small fluctuations are persistent and those of large fluctuations are anti-persistent in the short term, while the cross-correlations of all kinds of fluctuations are persistent in the long term, and also find the multi-fractality of cross-correlation between stock markets in China and Japan are stronger than others. P. Pavón-Domínguez, et al. [2] find the price series of livestock products are of a multi-fractal nature., and he main source of multi-fractality is the broadness of the probability function. Shu-Peng Chen, et al. [3] investigated the nonlinear dynamical mechanisms in China's agricultural futures markets, confirm the existence of multi-fractal features, and identified that long-range correlation mechanism constitutes major contributions in the formation in the multi-fractals of the markets. Meysam Bolgorian, et al. [4] investigated the multi-fractal properties of the trading behavior of individual and institutional traders in the Tehran Stock Exchange (TSE), compare the results with S\&P 500, conclude that long-range correlation is the main source of multi-fractality for TSE while fat-tailed probability distribution for S\&P 500. Xinsheng Lu, et al. [5] studies the multi-fractal properties of the Chinese stock index futures market, using ten-minute closing prices, find the multi-fractality is mainly due to long-range correlations. Yin Yuan, et al. [6] studied the Shanghai and Shenzhen stock index in China, find significant multi- fractal features exist in both markets, and Shenzhen Index has a stronger persistence and greater risk, long-term correlation is the main cause of multi-fractal. Lin $\mathrm{Xu}$, et al. [7] combined the MF-DFA and the sliding window technique, analyze the daily return series of CITIC's six kinds of pure stock-style asset index, find all the indices have multi-fractal character, and long-range correlation is the main factor in the formation of multi-fractal. Ling-Yun He, et al. [8] investigated the multi-fractality and its underlying formation mechanisms in international crude oil markets (Brent and WTI), and find both markets exhibit multi-fractal properties. Weijie Zhou, et al. [9] apply the MFDMA (multi-fractal detrended moving average) to investigate and compare the efficiency and multifractality of 5-min high-frequency China Securities Index 300 (CSI 300), find that the CSI 300 market becomes closer to weak-form efficiency after the introduction of CSI 300 future, while extreme events and fat-distribution are the main origin of multi-fractality. Miguel A. Rivera-Castro, et al. [10] extends the MF-DFA method and propose the Asymmetric Detrended Fluctuation Analysis (A-DFA) method. 
Guangxi Cao, et al. [11] utilize the A-MFDAF method to examine the asymmetric multi-fractal scaling behavior of Chinese stock markets with uptrends or downtrends, find the multi-fractality degree of Chinese stock markets with uptrends is stronger than with downtrends, correlation asymmetries are more evident in large fluctuations than in small fluctuations, multi-fractality is related to long-range correlations when the market is going up, whereas it is related to fat-tailed distribution when the market is going down. Jose Alvarez-Ramirez, et al. [12] explore the existence of asymmetries in the scaling behavior of time-series based on A-DFA, and find the asymmetries are scale-dependent, meaning that for some scales the scaling behavior is symmetric but asymmetric for another time scales. Syed Aun R. Rizvi, et al. [13] investigated 22 broad market indices, find the empirical evidence of the impact of the 'stage of market development' on the efficiency of the market.

In addition, recently there are some MF-DFA based studies in other fields, for example, Rosilda B. de Benicio, et al. [14] examine statistical properties of a daily hot pixel time series recorded in Brazil during the period 1998-2006, confirm the multi-fractal behavior of hot pixel dynamics. M Sadegh Movahed, et al. investigate the sunspot number fluctuations, and conclude that the multi-fractal nature is almost entirely due to long range correlations. H.J. Tanna, et al. [16] study the Earth's ionospheric scintillation index time series, prove that the multi-fractal mainly comes from long-range correlation. Jing Wang, et al. [17] investigate the traffic signals, proves the traffic signal has multi-fractal properties, and the property is dependent on the selected time scale. $\mathrm{Zu}-\mathrm{Guo} \mathrm{Yu}$, et al. [18] study the daily rainfall in the Pearl River Basin, and confirm the existence of multi-fractal properties.

The above studies on financial markets mainly focus on one unique market index, to confirm the existence of multi-fractal properties, there have been few studies comparing the characteristics of different markets. Therefore, this paper divides several indices into different groups according to geographical locations, respectively confirm the existence of multi-fractal properties, then compare whether the source of multi-fractal properties of indices from various regions have differences.

\section{MF-DFA method}

Let $\mathrm{X}(\mathrm{t})$ be a time series, $\mathrm{t}=1,2 \ldots \mathrm{N}, \mathrm{N}$ is the length of the time series, the process of MF-DFA is as follows:

$$
\text { Construct a new time series } Y=\sum_{t=1}^{j}(x(\mathrm{t})-\bar{x}), \mathrm{i}=1,2, \ldots \mathrm{N}, \bar{x}=\frac{1}{N} \sum_{t=1}^{N} x(\mathrm{t})
$$

Divide the original time series $\mathrm{X}$ and the new series $\mathrm{Y}$ into $N_{S}=\operatorname{int}(N / n)$ none-overlapping parts, the length of each part is $n$. Since $N$ is not necessarily integer multiples of $n$, in order to ensure no data is lost, the time series is divided once again from the end, so 2Ns subsequences are obtained. Let $X_{i, j}$ be the $j$-th element in the $i$-th sub module of the original series $X(t)$, let $Y_{i, j}$ be the $j$-th element in the $\mathrm{i}$-th sub module of the new series $\mathrm{Y}(\mathrm{t})$, then the corresponding relationship between $\mathrm{X}_{\mathrm{i}, \mathrm{j}}$ and the original series as well as $Y_{i, j}$ and the new series is as follows:

$$
\begin{aligned}
& X_{i, j}=X((i-1) n+j), Y_{i, j}=Y((i-1) n+j), i=1,2, \ldots N s \\
& X_{i, j}=X(N-(i-N s) n+j), Y_{i, j}=Y(N-(i-N s) n+j), i=N s+1, \ldots, 2 N s
\end{aligned}
$$

The length $n$ of each data ranges from 5 to N/4 [19].

3. For each sub series $Y_{i}$ in the newly constructed time series $Y$, compute its least squares fitting curve $L_{i}$, let the $x$-axis be 1 -n (the length of sub series), then compute the fluctuation function for each interval.

$$
F_{i}(\mathrm{n})=\frac{1}{n} \sum_{k=1}^{n}\left(\mathrm{y}_{\mathrm{i}, \mathrm{k}}-\mathrm{L}_{Y_{i}}(\mathrm{k})\right)^{2}, \mathrm{i}=1,2 \ldots 2 \mathrm{Ns}
$$

Then extend it to the q-order fluctuation function for the entire series.

$$
F_{q}(n)=\left(\frac{1}{2 N_{S}} \sum_{i=1}^{2 N_{S}}\left[F_{i}(n)\right]^{q / 2}\right)^{1 / q}
$$


Let the order $\mathrm{q}$ be the integers from -10 to 10 , for each q, analyze the one-order function of $\log$ $(\mathrm{Fq}(\mathrm{n}))$ and $\log (\mathrm{n})$, the slope is the q-order generalized Hurst exponent $\mathrm{H}(\mathrm{q})$. If $\mathrm{H}(\mathrm{q})$ is constant, the series is mono-fractal, if $\mathrm{H}(\mathrm{q})$ is the function of $\mathrm{q}$, then the series is multi-fractal.

If $\mathrm{H}(\mathrm{q})>0.5$, the correlation in the time series is persistent, means the rise of price have a large probability of being followed by another rise, the fall of price have a large probability of following by another fall. If $\mathrm{H}(\mathrm{q})<0.5$, then the correlations in the time series is anti-persistent, means the rise of price have a large probability of being followed by a fall, and vice versa. If $H(q)=0.5$, only short-run correlations or no correlations exist in the series.

Then we use the Hurst exponent to calculate Renyi exponent $\tau(\mathrm{q})$,

$\mathrm{T}(\mathrm{q})=\mathrm{qH}(\mathrm{q})-1$

Then get the singularity exponent and multi-fractal spectrum $\mathrm{f}(\mathrm{a})$ through the Legendre transform.

$\mathrm{a}=\mathrm{H}(\mathrm{q})+\mathrm{qH}(\mathrm{q})$

$\mathrm{f}(\mathrm{a})=\mathrm{q}[\mathrm{a}-\mathrm{h}(\mathrm{q})]+1$

The singularity exponent a can depict the singularity level in a complex system, the larger the exponent is, the smaller the singularity is. The multi-fractal spectrum $f$ (a) can reflect the fractal dimension of singularity exponent $a$. The width of $f(a)$ is $a_{\max }-a_{\min }$, the larger the width value is, the more irregular the time series distribution is, and the stronger the multi-fractal strength is.

\section{Data source}

This paper selects several stock market indices according to geographical locations. The indices are Shanghai Composite Index, Shenzhen Composite Index, Nikkei 225 Index, Hang Seng Index, and Singapore Straits Times from Asia, and S\&P 500, Dow Jones, Nasdaq, Canada S\&P TSX, Mexico MXX from America, as well as Germany DAX30, England FTSE 100, France CAC40, Italy FTMIB from Europe. The data range of each index is from September 1, 2006 to September 30, 2013, all data is from the Bloomberg database.

Table 1 Hurst exponents of main Asian stock markets indices

\begin{tabular}{ccccccccccc}
\hline \multirow{2}{*}{$\mathrm{q}$} & \multicolumn{2}{c}{ Shanghai Composite } & \multicolumn{2}{c}{ Shenzhen Composite } & \multicolumn{2}{c}{ Nikkei 225} & \multicolumn{2}{c}{ Hang Seng } & \multicolumn{3}{c}{ Singapore Straits Times } \\
\cline { 2 - 11 } & original & new & original & new & original & new & original & new & original & new \\
\hline-10 & 0.7015 & 0.7881 & 0.7734 & 0.7444 & 0.7300 & 0.7230 & 0.7964 & 0.8025 & 0.7778 & 0.8129 \\
-9 & 0.6848 & 0.7760 & 0.7640 & 0.7339 & 0.7212 & 0.7133 & 0.7860 & 0.7905 & 0.7675 & 0.8024 \\
-8 & 0.6722 & 0.7612 & 0.7528 & 0.7213 & 0.7110 & 0.7017 & 0.7732 & 0.7759 & 0.7553 & 0.7898 \\
-7 & 0.6564 & 0.7431 & 0.7394 & 0.7062 & 0.6988 & 0.6879 & 0.7575 & 0.7580 & 0.7407 & 0.7745 \\
-6 & 0.6392 & 0.7209 & 0.7231 & 0.6878 & 0.6845 & 0.6712 & 0.7377 & 0.7360 & 0.7234 & 0.7557 \\
-5 & 0.6261 & 0.6936 & 0.7031 & 0.6655 & 0.6676 & 0.6512 & 0.7130 & 0.7092 & 0.7030 & 0.7331 \\
-4 & 0.6146 & 0.6602 & 0.6782 & 0.6382 & 0.6479 & 0.6280 & 0.6830 & 0.6777 & 0.6794 & 0.7065 \\
-3 & 0.6059 & 0.6201 & 0.6491 & 0.6053 & 0.6262 & 0.6024 & 0.6494 & 0.6431 & 0.6530 & 0.6773 \\
-2 & 0.5954 & 0.5763 & 0.6209 & 0.5706 & 0.6043 & 0.5763 & 0.6170 & 0.6093 & 0.6269 & 0.6473 \\
-1 & 0.5845 & 0.5379 & 0.5987 & 0.5438 & 0.5834 & 0.5510 & 0.5893 & 0.5795 & 0.6064 & 0.6177 \\
0 & 0.5727 & 0.5066 & 0.5817 & 0.5240 & 0.5623 & 0.5262 & 0.5650 & 0.5538 & 0.5925 & 0.5889 \\
1 & 0.5609 & 0.4797 & 0.5673 & 0.5081 & 0.5372 & 0.5014 & 0.5403 & 0.5305 & 0.5825 & 0.5610 \\
2 & 0.5432 & 0.4565 & 0.5538 & 0.4956 & 0.5015 & 0.4762 & 0.5092 & 0.5089 & 0.5706 & 0.5341 \\
3 & 0.5244 & 0.4361 & 0.5407 & 0.4854 & 0.4551 & 0.4513 & 0.4695 & 0.4888 & 0.5526 & 0.5088 \\
4 & 0.5055 & 0.4182 & 0.5283 & 0.4769 & 0.4096 & 0.4281 & 0.4275 & 0.4708 & 0.5306 & 0.4855 \\
5 & 0.4874 & 0.4026 & 0.5168 & 0.4694 & 0.3733 & 0.4076 & 0.3909 & 0.4550 & 0.5087 & 0.4649 \\
6 & 0.4708 & 0.3892 & 0.5065 & 0.4627 & 0.3464 & 0.3903 & 0.3616 & 0.4416 & 0.4893 & 0.4470 \\
7 & 0.4561 & 0.3776 & 0.4973 & 0.4566 & 0.3264 & 0.3759 & 0.3388 & 0.4303 & 0.4730 & 0.4319 \\
8 & 0.4433 & 0.3677 & 0.4892 & 0.4510 & 0.3111 & 0.3640 & 0.3209 & 0.4207 & 0.4594 & 0.4192 \\
9 & 0.4324 & 0.3591 & 0.482 & 0.4459 & 0.2991 & 0.3542 & 0.3066 & 0.4127 & 0.4482 & 0.4086 \\
10 & 0.4231 & 0.3517 & 0.4756 & 0.4411 & 0.2894 & 0.3459 & 0.2951 & 0.4058 & 0.4388 & 0.3996 \\
$\Delta \mathrm{h}$ & 0.2784 & 0.4364 & 0.2978 & 0.3033 & 0.4406 & 0.3771 & 0.5013 & 0.3967 & 0.3390 & 0.4133 \\
\hline
\end{tabular}




\section{Empirical analysis}

Each index is peaked (kurtosis greater than 3) and their skewness is not 0, so all the indices are not normally distributed. Then we use the method above to calculate each index's Hurst exponent and analyze the source of multi-fractal. There are two main sources of multi-fractal in the time series: long-range volatility correlations and fat-tail probability distribution. To measure the contribution extent of the two factors, this paper rearrange the raw data of each index, to get a new series of corresponding rearranged data. The rearranged data can keep the overall distribution character while destroy the long-range correlations of the series, that is to say, the rearranged data and original data have the same distribution but different long-range correlations, so we can compare the character of the original data and the rearranged data's Hurst exponent, to analyze the contribution extent of the two factors.

To ensure the data is sufficiently disrupted and rearranged, this paper runs $100 \mathrm{~N}$ time operation for each original index, $\mathrm{N}$ is the length of the corresponding index, each time randomly switch two elements in the series, and form a new random series. Then set the value of order q from -10 to 10 , and calculate the Hurst exponents of each Asian stock market index's original and rearranged data, as shown in Table 1.

As can be seen from Table 1, when the order q changes from -10 to 10, each index's Hurst exponent is obviously not constant, indicating that these indices all present multi-fractal features. And as q becomes larger, the Hurst exponent becomes smaller. When q is negative or small positive number, most Asian indices' $\mathrm{h}(\mathrm{q})>0.5$ showing persistence character, while when $\mathrm{q}$ is a relatively large positive number, $\mathrm{h}(\mathrm{q})<0.5$, showing anti- persistence character. And the q values at which each index turn from persistent to anti-persistent are different, for example, the q value for Shanghai Composite that turn from persistent to anti-persistent is 4, while Shenzhen Composite is 6, Nikkei 225 is 2, Hang Seng is 2, Singapore Straits Times is 5. Among these Asian indices, Hong Kong's Hang Seng Index has the maximum $\Delta \mathrm{h}$, indicating it has the most obvious multi-fractal feature and the greatest risk.

Then we observe the source of multi-fractal through observing each index's original series and rearranged series. As described above, the rearranged series has disrupted long-range correlations of the original time series, therefore, if the difference between original series' Hurst exponents and rearranged series' Hurst exponents is small, the multi-fractal property is mainly caused by the fat-tailed probability distribution, if the difference is relatively big, indicating that long-range correlation's contribution is more significant.

For most Asian indices, the difference between two series' Hurst exponents is small when order q is negative or relatively small positive numbers, the multi-fractal is mainly caused by the fat-tailed probability distribution. While the difference between two series' Hurst exponents is big when order $\mathrm{q}$ is relatively large positive numbers, the multi-fractal is mainly caused by long-range correlation.

Similarly, we compute the Hurst exponents for each American indices, and find that major American indices also show a multi-fractal feature, the same as major Asian stock indices. And h (q) also decreases with the increasing of $\mathrm{q}$, and all the indices show persistence feature when $\mathrm{q} \leq 1$, anti-persistence when $\mathrm{q}>1$. Among all the American major indices, Dow Jones index has the maximum $\Delta \mathrm{h}$, indicating it has the most obvious multi-fractal feature and the greatest risk. When order q is negative or relatively small positive numbers, the difference between most American stock indices' original series' and rearranged series' Hurst exponents is big, the multi-fractal is mainly caused by the long-range correlation. While when q is relatively big positive number, the difference between the two series' Hurst exponents is relatively small, the multi-fractal is mainly caused by fat-tailed probability distribution. This phenomenon is opposite to the situation in Asia.

Similarly, we compute the Hurst exponents of each European indices, and find major European stock indices also show multi-fractal features, $h(q)$ also decreases with the increase of $q$, and all the indices show persistence feature when order $\mathrm{q}$ is negative or relatively small positive numbers, and anti-persistence feature when $\mathrm{q}>1$. Among all the indices, Britain FTSE 100 index has the maximum $\Delta \mathrm{h}$, the most obvious multi-fractal feature and the greatest risk. For European stock indices, there is 
little difference when order $\mathrm{q}$ is either negative or positive. The Hurst exponents of original series and rearranged series almost remain the same distance, indicating that the contribution to multi-fractal from the long-range correlations and fat-tail probability distribution are almost the same.

\section{Conclusion}

In this paper, we respectively select several representative stock indices in Asia, America and Europe continent, compute their Hurst exponents and draw the following conclusions:

Through the computation of Hurst exponents, we confirm the existences of multi-fractal feature of each index, and it is not suitable for using traditional efficient market theory to study the various indices.

When order $q$ is negative or a relative small positive number, each index's $h(q)>0.5$, showing persistence feature, while when order q is a relatively big positive number, each index's $h(q)<0.5$, showing anti-persistence feature,

Based on the $\Delta \mathrm{h}$ value analysis, the index which has the largest value and the most obvious multi-fractal feature as well as the greatest risk in Asia is Hong Kong's Hang Seng Index, in America is Dow Jones Index, while in Europe is FTSE 100 index.

Through rearranging the data and the analysis of the source of multi-fractal, we find that

For most of the Asian indices, there is little difference between the Hurst exponents of original series and the rearranged series when order $\mathrm{q}$ is negative or a small positive number, the multi-fractal feature is mainly caused by fat-tailed probability distribution. While the difference between Hurst exponents increases when $\mathrm{q}$ is a relatively big positive number, the multi-fractal feature is mainly caused by long-range correlations.

The situation of American indices is just the opposite of Asian indices, when order q is negative or a small positive number, the multi-fractal feature is mainly caused by long-range correlations, while when q is a relatively big positive number, the multi-fractal feature is mainly caused by fat-tailed probability distribution.

As European indices, for any order q value, the contribution to multi-fractal from long-range correlations and fat-tail probability distribution is approximately the same.

\section{Acknowledgment}

This work is partially supported by the National Natural Science Foundation of China through the Operational Program "Support Model Integration Principles and Methods of Unconventional Emergency Response Decision Mission Planning" (Grant No. 91024028) as well as the Operational Program "Research on the measuring methods of two -side customer relationship value of platform enterprises” (Grant No. 71172156).

\section{References}

[1] Ma, Feng, Yu Wei, and Dengshi Huang, Multifractal detrended cross-correlation analysis between the Chinese stock market and surrounding stock markets, Physica A: Statistical Mechanics and its Applications 392 (7) (2013) p.1659-1670.

[2] Pavón-Domínguez, P., et al., Multifractal detrended fluctuation analysis of sheep livestock prices in origin, Physica A: Statistical Mechanics and its Applications 392 (19) (2013) p. 4466-4476.

[3] Chen, Shu-Peng, Ling-Yun He, Multifractal spectrum analysis of nonlinear dynamical mechanisms in China's agricultural futures markets, Physica A: Statistical Mechanics and its Applications 389 (7) (2010) p.1434-1444.

[4] Bolgorian, Meysam, Reza Raei, A multifractal detrended fluctuation analysis of trading behavior of individual and institutional traders in Tehran stock market, Physica A: Statistical Mechanics and its Applications 390 (21) (2011)p. 3815-3825. 
[5] Lu, Xinsheng, et al., Multifractal detrended fluctuation analysis of the Chinese stock index futures market, Physica A: Statistical Mechanics and its Applications 392 (6) (2013) p.1452-1458.

[6] Ying Yuan, Xintian Zhuang, Xiu Jin, Analysis of the Chinese stock market's multi-scale characteristics and its causes based on MF-DFA, Journal of Industrial Engineering and Engineering Management 23 (4) (2009)p. 96-99.

[7] Lin Xu, Guanghui Song, Wenwei Guo, Multifractal analysis on stock style asset returns based on sliding windows MF-DFA, Systems Engineering-theory \& Practice 32 (9) (2012) p.1891-1899.

[8] He, Ling-Yun, Shu-Peng Chen, Are crude oil markets multifractal? Evidence from MF-DFA and MF-SSA perspectives, Physica A: Statistical Mechanics and its Applications 389 (16) (2010) p.3218-3229.

[9] Zhou, Weijie, Yaoguo Dang, Rongbao Gu, Efficiency and multifractality analysis of CSI 300 based on multifractal detrending moving average algorithm, Physica A: Statistical Mechanics and its Applications 392 (6) (2013) p.1429-1438.

[10] Rivera-Castro, Miguel A., et al., Detecting switching points using asymmetric detrended fluctuation analysis, Physica A: Statistical Mechanics and its Applications 391 (1) (2012) p.170-179.

[11] Cao, Guangxi, Jie Cao, Longbing Xu, Asymmetric multifractal scaling behavior in the Chinese stock market: Based on asymmetric MF-DFA, Physica A: Statistical Mechanics and its Applications 392 (4) (2013) p.797-807.

[12] Alvarez-Ramirez, Jose, Eduardo Rodriguez, Juan Carlos Echeverria, A DFA approach for assessing asymmetric correlations, Physica A: Statistical Mechanics and its Applications 388 (12) (2009) 2263-2270.

[13] Rizvi, Syed Aun R., et al., An analysis of stock market efficiency: Developed vs Islamic stock markets using MF-DFA, Physica A: Statistical Mechanics and its Applications 407 (2014):p. 86-99.

[14] de Benicio, Rosilda B., et al., Multifractal behavior of wild-land and forest fire time series in Brazil, Physica A: Statistical Mechanics and its Applications 392 (24) (2013) p.6367-6374.

[15] Movahed, M. Sadegh, et al., Multifractal detrended fluctuation analysis of sunspot time series, Journal of Statistical Mechanics: Theory and Experiment 2006 (2) (2006) P02003.

[16] Tanna, H. J., and K. N. Pathak, Multifractality due to long-range correlation in the L-band ionospheric scintillation S 4 index time series, Astrophysics and Space Science 350 (1) (2014) p.47-56.

[17] Wang, Jing, Pengjian Shang, Xingran Cui. Multiscale multifractal analysis of traffic signals to uncover richer structures, Physical Review E 89 (3) (2014) 032916.

[18] Yu, Zu-Guo, et al., Multifractal analyses of daily rainfall time series in Pearl River basin of China, Physica A: Statistical Mechanics and its Applications 405 (2014) p.193-202.

[19] C. K. Peng, S.V. Buldyrev, S. Havlin, et al., Mosaic organization of DNA nucleotides, Physical Review E 49 (1994) p.1685-1689. 